\title{
Profile of stress among a group of female school children in their early adolescence in the Galle educational zone; a descriptive cross sectional study
}

\author{
Madhushanthi HJ ${ }^{1}$, Wimalasekera SW ${ }^{2}$, Goonewardena CSE ${ }^{2}$, AmarasekaraAATD ${ }^{3}$, Lenora $\mathbf{J}^{4}$ \\ ${ }^{I}$ Faculty of Allied Health Sciences, University of Ruhuna, Galle, Sri Lanka. \\ ${ }^{2}$ Faculty of Medical Sciences, University of Sri Jayewardenepura, Nugegoda, Sri Lanka. \\ ${ }^{3}$ Faculty of Allied Health Sciences, University of Sri Jayewardenepura, Nugegoda, Sri Lanka. \\ ${ }^{4}$ Faculty of Medicine, University of Ruhuna, Galle, Sri Lanka.
}

Correspondence: Ms. H. J.H. Madhushanthi

e-mail: madhushani122@gmail.com

(D) https://orcid.org/0000-0002-8732-9102

Submitted on 02.01.2021 and accepted for publication on 16.06.2021

\begin{abstract}
Introduction: Age ranging from 11 to 14 years is regarded as early adolescence. Females are particularly vulnerable to experience stress with rapid physical, psychological, cognitive and social changes accompanied in adolescence. Stress experienced in adolescence is linked with a range of adverse physical and psychological consequences. The study aimed to determine the level of stress and factors contributing to stress among female adolescents in the Galle educational zone, Sri Lanka.
\end{abstract}

Methods: A school based cross - sectional study was conducted on female adolescents (11 - 14 years, $n=218$ ). A self-administered socio-demographic questionnaire and psychosocial adversity scale were used to assess socioeconomic status and psychosocial adversities respectively. The Body Mass Index (BMI), waist hip ratio (WHR), waist height ratio (WHtR) and fat percentage (FM\%) were calculated to assess the nutritional status. Adolescent Stress Questionnaire (ASQ), which measures 9 dimensions of stress, was used to assess stress.

Results: Normal nutritional status was observed in 118 (54.1\%) while 65 (29.8\%) and 35(16.1\%) adolescents were underweight and overweight respectively. A majority of adolescents $(n=158,72.9 \%)$ were experiencing stress. An increased stress levels were observed for the domains of school attendance $(n=203,93.1 \%)$, future uncertainty $(n=202,92.7 \%)$, school performance $(n=185,84.9 \%)$, emerging responsibility $(n=186,85.3 \%)$, and conflict of school/leisure time $(n=178,81.7 \%)$.

Conclusions: Most adolescents considered future uncertainty and school attendance as the greatest stress inducing factors. Urgent measures to decrease stress levels amongst these adolescents need to be implemented.

Key words: Early female adolescents, profile of stress.

\section{Introduction}

Transition from care dependent childhood to care independent adulthood is adolescence. According to the WHO, age 11 to 14 years is regarded as early adolescence and this period is characterised by significant development in physical, psychological and social domains (1). Generally cortisol level increases in female adolescents with the onset of puberty due to an elevated level of gonadal hormones (2). Therefore female adolescents are particularly vulnerable for stressors possibly due to interaction between glucocorticoids and gonadal hormones. 
Alternatively there are differences between genders in stress hormone activation, in particular female adolescents demonstrate greater stress hormone reactivity compared to male adolescents (3). The brain continues to mature, particularly the subcortical structures of the brain, which are highly involved in emotional and cognitive development that, undergo marked structural and functional changes during the adolescence. Recent human and nonhuman studies have discovered, stress can alter the neural architecture within the corticolimbic regions of developing adolescent brain, thus it could negatively impact on cognitive development and academic outcomes too (4). Therefore, taken together stress experiences in the adolescence lead to greater exposure of the brain to steroid hormones producing long lasting effects on developing brain.

Stress experience in adolescence is more likely to link with a range of adverse physical and psychological consequences such as substance abuse, drug abuse, poisoning, physical inactivity and uncontrolled weight gain. Survey of previous literature revealed that depression and anxiety are more prevalent among female adolescents than male adolescents in Sri Lanka $(5,6)$. However studies on the factors associated with stress among Asian female adolescents are limited. Considering these facts, the present study was aimed to determine stress profile of female adolescents.

\section{Methods}

Study population and Design: A school-based descriptive cross-sectional study was conducted on randomly selected schools in the Galle educational zone in Sri Lanka. Female adolescents aged 11- 14 years $(n=218)$ residing in the city of Galle were recruited. Schools were randomly selected representing all categories of schools classified by the Ministry of Education. The classes were randomly selected covering age 11 to 14 years. All students in each selected class were recruited.

\section{Assessment of socioeconomic status and psycho- social adversities}

A self-administered socio-demographic question-naire was used to assess the educational level, employment status and income level of the parents.
Parents' occupation was categorised based on criteria of the Hollingshead Index of Occupation Scale matched as closely as possible to modern occupations (7). Income to need ratio was calculated, by dividing total family income by official poverty threshold for a family of that size. Official poverty threshold published by the department of Census and Statistics, Sri Lanka was applied to calculate income to need ratio. Finally family socioeconomic status (SES) composite score (SES index) was derived by standardizing and averaging income to need ratio, parental education and Hollingshead occupation scale rank.

Psychosocially adverse factors were objectively assessed using the psychosocial adversity scale and this scale has been widely used in child development studies in Asian settings (8). It assesses the home environment and the parent's health status. Psychosocial adversity index (PA index) score was derived summing the total scores. Higher scores indicated higher levels of psychosocial adversity. Items were scored by interviewing the adolescent.

Adolescents' stress was assessed using Adolescence Stress Questionnaire (ASQ) that has been widely used to measure stress of adolescence $(9,10,11)$. It has been validated to Sinhala language and showed acceptable internal reliability and construct validity (12). The questionnaire measures nine dimensions of adolescence stress including stress of home life, school performance, school attendance, romantic relationships, peer pressure, teacher interaction, future uncertainty, school/ leisure conflict and emerging adult responsibility. It is a self-administered questionnaire and each item was rated on a 5 point Likert scale where $1=$ not at all stressful/ irrelevant to me, $2=$ a little stressful, $3=$ moderately stressful, $4=$ quite stressful and $5=$ very stressful. The marks for answers ranked from 1 - 5 score and total score for each component scale were calculated by the counting the scores of the items that belong to the scale. The score more than 3 per each domain was considered as the cut-off level to indicate stress. Adolescents with a total score of more than 156 (out of 250) were categorised into the stressed group. 


\section{Assessment of anthropometric measurements}

In order to assess nutritional status of study participants, body mass index (BMI), fat mass percentage (FM\%), waist circumference (WC), hip circumference (HC), waist hip ratio (WHR) and waist height ratio (WHtR) were calculated. Height for age $\mathrm{z}$ score (HAZ) and weight for age $\mathrm{z}$ score (WAZ) were calculated using WHO sex specific growth reference values for adolescents. Triceps and subscapular skin fold thickness were measured using skin fold caliper according to standard protocol and $\mathrm{FM} \%$ was calculated using a validated FM\% estimation equation developed for Sri Lankan children by Wickramasinghe et al. (13).

Data were analysed using SPSS 20 version software. Descriptive statistics were used to describe nutritional and psychosocial status characteristics. Independent sample t-test was applied to compare means between stressed and non-stressed groups. P value less than 0.05 was considered as statistically significant. Ethical approval was granted by the Ethics Review Committee, Faculty of Medical Sciences, the University of Sri Jayewardenepura. Informed written consent from the parents and assent of the child were taken prior to the commencement of data collection.

\section{Results}

The age of the study participants ranged from 11 to 14 years with mean age $12.31( \pm 1.16)$ years. Majority of the participants were Sinhalese by ethnicity and Buddhist by religion. About $49.5 \%$ of adolescents had two or three siblings. Most (57\%) of the adolescents had attained menarche by the time of the study. Majority of adolescents $(\mathrm{n}=143,68.3 \%)$ were in nuclear families while others were living in extended families. Around half of the study participants (54\%) resided in semi urban areas.

Mean BMI of the study sample was $18.22( \pm 4.19)$. Study participants were categorised into under-weight, normal and overweight groups based on age and sex specific BMI percentile values based on the WHO growth reference values.
Accordingly, 29.8\% were underweight among female adolescents, while $54.1 \%$ and $16.1 \%$ were within normal and overweight categories. Around $35 \%(n=77)$ of female adolescents were centrally obese in this sample. Female adolescents undergo significant physical changes in body fat distribution and muscle mass with the onset of puberty. However there was no statistically significant difference in body fat distribution between post pubertal adolescents and pre pubertal adolescents in this study group $(p>0.05)$.

Majority of the participants $(\mathrm{n}=158,72.5 \%)$ belonged to the stressed group whereas others $(\mathrm{n}=60,27.5 \%)$ were classified as the non-stressed groups. As the results tabulated in Table 1, age and nutritional parameters were not significantly different between stressed and non-stressed groups. Moreover, socioeconomic index and psychosocial adversity index were also not different between the two groups $(p>0.05)$. This confirms that both groups are similar other than for the level of stress.

The ASQ score ranged from 83 to 208 , and the mean stress score of these adolescents was 145.4 \pm 24.3. The factors contributing to stress among female adolescents are summarised in Table 2. Most adolescents reported the stress of future uncertainty $(\mathrm{n}=202,92.7 \%)$ and school attendance $(\mathrm{n}=203,93.1 \%)$ as the greatest inducing factors. Other important factors responsible for their stress were school performance, emerging responsibilities, conflict between school/ leisure time, romantic relationships, peer pressure and teacher interactions. Pressure from the parents to perform better in school made a considerable contribution $(n=180$, $82.5 \%$ ) to stress associated with school performance. The scores for having too much home work $(\mathrm{n}=186,85.3 \%)$, too much expectation by parents from adolescents $(\mathrm{n}=185,84.8 \%)$ and not having enough time for leisure activities $(\mathrm{n}=178,81.6 \%)$ were significantly higher amongst the stressed group compared to the non-stressed group $(p<0.05$ for all). 
Table 1: Anthropometric measures, SES index score and psychosocial adversity index score among adolescents

\begin{tabular}{lccccc}
\hline \multirow{2}{*}{ Characteristics } & \multicolumn{2}{c}{$\begin{array}{c}\text { Stressed group } \\
(\mathbf{n}=\mathbf{1 5 8})\end{array}$} & $\begin{array}{c}\text { Non-stressed group } \\
(\mathbf{n}=\mathbf{6 0})\end{array}$ & $\boldsymbol{p}$ value \\
\cline { 2 - 5 } & \multicolumn{2}{c}{ Mean (SD) } & \multicolumn{2}{c}{ Mean (SD) } & \\
\hline Age & 12.3 & $(1.1)$ & 12.2 & $(1.1)$ & 0.825 \\
BMI & 18.2 & $(4.1)$ & 18.0 & $(4.3)$ & 0.763 \\
Fat percentage & 21.0 & $(3.9)$ & 21.2 & $(4.1)$ & 0.863 \\
Waist circumference & 64.0 & $(8.6)$ & 64.3 & $(8.5)$ & 0.770 \\
Hip circumference & 78.3 & $(10.2)$ & 77.0 & $(9.8)$ & 0.393 \\
Waist hip ratio & 0.8 & $(0.05)$ & 0.8 & $(0.04)$ & 0.200 \\
Waist height ratio & 0.43 & $(0.09)$ & 0.4 & $(0.05)$ & 0.916 \\
SES index & 32.0 & $(11.8)$ & 30.6 & $(11.3)$ & 0.465 \\
Psychosocial adversity index & 3.0 & $(2.6)$ & 3.4 & $(2.6)$ & 0.298 \\
\hline
\end{tabular}

Table 2: Level of stress for each dimension in the ASQ experienced among adolescents

\begin{tabular}{lllll}
\hline Component in the ASQ & \multicolumn{2}{c}{ Stressful } & \multicolumn{2}{c}{ Non-stressful } \\
& $\mathbf{n}$ & $\mathbf{( \% )}$ & $\mathbf{n}$ & $\mathbf{( \% )}$ \\
\hline Stress of - home life & 133 & $(61 \%)$ & 85 & $(39 \%)$ \\
- school performance & 185 & $(84.9 \%)$ & 33 & $(15.1 \%)$ \\
- school attendance & 203 & $(93.1 \%)$ & 15 & $(11.5 \%)$ \\
- romantic relationship & 141 & $(64.7 \%)$ & 77 & $(35.3 \%)$ \\
- peer pressure & 133 & $(61 \%)$ & 85 & $(39 \%)$ \\
- teacher interaction & 131 & $(60.1 \%)$ & 87 & $(39.9 \%)$ \\
- future uncertainty & 202 & $(92.7 \%)$ & 16 & $(7.3 \%)$ \\
- school / leisure time & 178 & $(81.7 \%)$ & 40 & $(1.3 \%)$ \\
- emerging responsibilities & 186 & $(85.3 \%)$ & 32 & $(14.7 \%)$ \\
\hline
\end{tabular}

\section{Discussion}

In this cross sectional study, it was found that an overwhelming number of female adolescents were stressed. This conforms with previous study findings high prevalence of stress among females $(9,11)$. A cross sectional survey conducted by Rodrigo and colleagues in the Rathnapura municipality indicate $36 \%$ and $28 \%$ of school students aged 14-18 years were diagnosed with depression and severe anxiety respectively (6). When considering the global data, it has been revealed that high proportion of female adolescents have experienced stress in India too (14). 
In addition, female adolescents exhibited higher perceived stress scores as measured by ASQ among European adolescents (11) and Norwegian adolescents (10) Adolescence is a window period in life which experiences substantial biological changes. The hormonal and physical changes of puberty are a stressful experience for most female adolescents as they have to adapt to the monthly menstruation. Therefore, reserved social life style experienced within the Sri Lankan cultural norms provides more opportunities for vulnerability to stressors.

Studying material with minimum interest and high teacher expectation, pressure of studies, attending school, compulsory school attendance, lack of respect from teachers and disagreement between teachers and student are significant factors contributing to stress among female adolescents in the present study. Rodrigo and colleagues explained examination related issues, difficulty in studying and problems with teachers as the major causative factors inducing depression and anxiety among adolescents. Other studies also observed harsh discipline by parents and teachers and violence observed at home leads to adverse mental health problems (15).

Conversely, not having enough leisure time, recreational activities and having too much homework create unnecessary psychological burden to adolescents. In Sri Lanka, education is provided free of charge to all students attending state schools. The current education system and school curriculum are highly exam-oriented and demand higher memorizing capacity, thus adolescents tend to be stressed within the competitive education environment. Students are confined into homework activities and it restricts their time to play and engage in recreational activities. Parental pressure to perform academically well to secure high scores at examinations and achieve other academic targets, lead to negative mental outcomes and undue stress amongst adolescents.

Academic study is a main possible source of stress of students in other Asian countries too, particularly India, Singapore, China and Korea $(16,17)$. Asian school aged adolescents tend to have more academic related stress compared to western countries. This difference might be attributed to differences in the two education systems and perceived value of education. As in Sri Lanka, frequent parental pressure to improve academic success and expectation and peer competition were the leading predisposing factors contributing to depression, anxiety and stress amongst Indian (14) and Chinese (18) adolescents. In addition, tuition class system has now been widely established, well popular and most students and parents have been victims of it. Unlike in the past, students tend to attend to classes to cover all subjects, parents pay high tuition fees in the hope of additional academic output regardless of vacation period.

There was no significant difference in stress of romantic relationship between stressed and nonstressed group in the present study. It is plausible that female adolescents are reluctant to explain their personal issues within the context of social and cultural taboo. However, most of the reported incidences of deliberate self-harm and suicide in adolescents in Sri Lanka are due to the problems related to romantic relationships (6).

Considering all these facts establishment of good teacher student and parent child relationship are important to secure trust and support to overcome anxiety and stress of adolescents. Provision of counselling programme at school and community level would help to ensure better psychological wellbeing. Moreover school authorities could implement parent awareness programmes focussing information on negative impact of parental pressure on academic success, importance of positive response and child friendly parenting style.

\section{Conclusions}

The findings provide clear evidence for the nature and extent of psychological problems encountered by early adolescent girls. It is clear that family, peer and teacher participation is vital in coping with stress. As such it is of paramount importance that school authorities and family members are aware and be responsive to the negative mental health outcomes associated with stress.

\section{Acknowledgement}

The study was funded by a university research grant of the University of Sri Jayewardenepura (ASP/06/RE/MED/2014/19). 


\section{References}

1. World Health Organization (2005). 'Nutrition in adolescence: issues and challenges for the health sector: issues in adolescent health and development', Geneva. Available from: http://www.who.int/iris/handle/10665/ 43342 (Accessed on 16 June 2017).

2. Lupien SJ, McEwen BS, Gunnar MR, Heim C. Effects of stress throughout the lifespan on the brain, behaviour and cognition. Nat Rev Neurosci. 2009; 10(6): 434.

3. Gunnar MR, Wewerka S, Frenn K, Long JD, Griggs C. Developmental changes in hypothalamus-pituitary-adrenal activity over the transition to adolescence: normative changes and associations with puberty. Development and Psychopathology. 2009; 21(1): 69-85.

4. Giedd JN, Rapoport JL. Structural MRI of pediatric brain development: what have we learned and where are we going? Neuron. 2010; 67(5); 728-734.

5. Perera H. Mental health of adolescent school children in Sri Lanka - a national survey. Sri Lanka Journal of Child Health. 2004; 33(3): 78-81.

6. Rodrigo C, Welgama S, Gurusinghe J, Wijeratne T, Jayananda G, Rajapakse S. Symptoms of anxiety and depression in adolescent students; a perspective from Sri Lanka. Child and Adolescent Psychiatry and Mental Health. 2010; 4(1): 10. DOI: 10.1186/1753-2000-4-10.

7. Hollingshead A. Four factor index of social status. Yale JSociol. 2011; 8: 21-52.

8. Bradley R. Using the HOME inventory to assess the family environment. Pediatr Nurs. 1988; 14(2); 97-102.

9. Byrne DG, Davenport SC, Mazanov J. Profiles of adolescent stress: The development of the adolescent stress questionnaire (ASQ). Journal of Adolescence. 2007; 30(3): 393-416.

10. Moksnes UK, Espnes GA. Evaluation of the Norwegian version of the Adolescent Stress Questionnaire (ASQ-N): Factorial validity across samples. Scandinavian Journal of Psychology, 2011; 52(6): 601-608.
11. De Vriendt T, Clays E, Moreno LA, Bergman P, VicenteRodriguez G, Nagy E, et al. Reliability and validity of the Adolescent Stress Questionnaire in a sample of European adolescents-the HELENA study. BMC Public Health. 2011; 11(1): 717. DOI: 10.1186/1471-2458-11-717.

12. Kathriarachchi LS, Hewage DC, Wimalasekera SW, Ranjan R, Mendis ALS. Home school environmental factors and peer pressure contributing to stress among adolescents in Colombo district in Sri Lanka - Preliminary results. Proceedings of South Asian Association of Physiologist Conference. (SAAPCON 2016), (2016); 229.

13. Wickramasinghe VP, Lamabadusuriya SP, Cleghorn GJ, Davies PS. Assessment of body composition in Sri Lankan children: validation of a skin fold thickness equation. Ceylon medical Journal. 2008; 53(3): 83-88. DOI: 10.4038/cmj.v53i3.247.

14. Bhasin SK, Sharma R, Saini NK. Depression, anxiety and stress among adolescent students belonging to affluent families: A school-based study. The Indian Journal of Pediatrics. 2010; 77(2): 161-165. DOI: 10.1007/s12098009-0260-5.

15. Miller KE, Jordans MJ. Determinants of children's mental health in war-torn settings: Translating research into action. Current Psychiatry Reports. 2016: 18(6); 58. DOI: 10.1007/s11920-016-0692-3.

16. Mitra C, Deb S. (2011) Mental and physical stress among students before board examinations and before admission to the next level: analyses of case studies. Paper presentation at the International conference on Students' Mental Health: Issues and Challenges, Puducherry.

17. Deb S, Strodl E, Sun J. Academic-related stress among private secondary school students in India. Asian Education and Development Studies. 2014;3(2); 118-134.

18. Zhao X, Zhu S, Ma, G. Comparison study on basic rights between Chinese, Japanese, Korean: American high school students. China Youth Study. 2009; 6: 1-7. 